\title{
A functional equation with Borel summable solutions and irregular singular solutions
}

\author{
By Sunao ŌuchI
}

(Received Apr. 18, 2016)

(Revised Sep. 29, 2016)

\begin{abstract}
A Functional equation $\sum_{i=1}^{m} a_{i}(z) u\left(\varphi_{i}(z)\right)=f(z)$ is considered. First we show the existence of solutions of formal power series. Second we study the homogeneous equation $(f(z) \equiv 0)$ and construct formal solutions containing exponential factors. Finally it is shown that there exists a genuine solution in a sector whose asymptotic expansion is a formal solution, by using the theory of Borel summability of formal power series. The equation has similar properties to those of irregular singular type in the theory of ordinary differential equations.
\end{abstract}

\section{Introduction.}

In this paper we study a functional equation

$$
\sum_{i=1}^{m} a_{i}(z) u\left(\varphi_{i}(z)\right)=f(z),
$$

where $\left\{a_{i}(z)\right\}_{i=1}^{m}(m \geq 2)$ and $f(z)$ are holomorphic in a sector with vertex $z=0$ or formal power series of $z$, and $\left\{\varphi_{i}(z)\right\}_{i=1}^{m}$ are holomorphic in a neighborhood of $z=0$ with $\varphi_{i}(0)=0$ and $\varphi_{1}^{\prime}(0)=\varphi_{2}^{\prime}(0)=\cdots=\varphi_{m}^{\prime}(0) \neq 0$. We study the existence of a solution of formal power series $\widetilde{u}(z)=\sum_{n=0}^{\infty} c_{n}^{0} z^{n}$. In case $\left\{a_{i}(z)\right\}_{i=1}^{m}$ are constants, it is studied in Ouchi [5]. Next we study the homogeneous case $f(z) \equiv 0$. We obtain the existence of a formal solution in the form of $\widetilde{v}(z)=e^{\psi(1 / z)} z^{\alpha} \widetilde{w}(z)$, where $\psi(t)$ is a polynomial of $t$ and $\widetilde{w}(z)=\sum_{n=0}^{\infty} c_{n}^{1} z^{n}$ with $\widetilde{w}(0)=1$, which is not studied in [5]. We also show under certain conditions of $\left\{a_{i}(z)\right\}_{i=1}^{m},\left\{\varphi_{i}(z)\right\}_{i=1}^{m}$ and $f(z)$ that there exists a Borel summable function $u(z)(w(z))$ in some sector such that $u(z) \sim \widetilde{u}(z)$ (resp. $w(z) \sim \widetilde{w}(z))$ asymptotically and $u(z)$ (resp. $\left.v(z)=e^{\psi(1 / z)} z^{\alpha} w(z)\right)$ satisfies (1.1).

We give notations and definitions. The set of all holomorphic functions on a region $U \subset \mathbb{C}$ is denoted by $\mathcal{O}(U)$. The set of all integers is denoted by $\mathbb{Z}$ and $\mathbb{Z}_{\geq 0}=\{n \in \mathbb{Z} ; n \geq$ $0\}$. Let $\theta \in \mathbb{R}$ and $R, \delta>0$. Define $S(\theta, \delta, R)=\{z ; 0<|z|<R ;|\arg z-\theta|<\delta\}$ and $S(\theta, \delta)=\{z ;|\arg z-\theta|<\delta\}$. We also define $S_{\{0\}}(\theta, \delta)=\{z \in S(\theta, \delta) ; 0<|z|<\rho(\arg z)\}$, where $\rho(\cdot)$ is some positive continuous function on $(\theta-\delta, \theta+\delta)$, which is a domain of sector type with vertex $z=0$. The set of all formal power series of $z$ is denoted by $\mathbb{C}[[z]]$. We define a subspace of $\mathbb{C}[[z]]$.

2010 Mathematics Subject Classification. Primary 30D05; Secondary 39B32, 44A10.

Key Words and Phrases. Borel summable, irregular singular, functional equation. 
Definition 1.1. Let $s \geq 0$. A formal power series $f(z)=\sum_{n=0}^{\infty} f_{n} z^{n}$ is said to be of Gevrey order $s$, if there exist constants $A, C \geq 0$ such that

$$
\left|f_{n}\right| \leq A C^{n} \Gamma(s n+1) \quad \text { for } \quad n \in \mathbb{Z}_{\geq 0} .
$$

We denote by $\mathbb{C}_{\{s\}}[[z]]$ the set of all formal power series of $z$ of Gevrey order $s$.

If $s=0, f(z)$ converges in a neighborhood of $z=0$ and the set of all convergent power series of $z$ is denoted by $\mathbb{C}\{z\}$.

\section{Formal solutions.}

Let

$$
L(z, u(z))=\sum_{i=1}^{m} a_{i}(z) u\left(\varphi_{i}(z)\right),
$$

where $\left\{\varphi_{i}(z)\right\}_{1 \leq i \leq m}(m \geq 2)$ are different holomorphic functions in a neighborhood of $z=0$ such that $\varphi_{i}(0)=0$ and

$$
\varphi_{1}^{\prime}(0)=\varphi_{2}^{\prime}(0)=\cdots=\varphi_{m}^{\prime}(0) \neq 0 .
$$

We may assume $\varphi_{i}^{\prime}(0)=1$ and $\varphi_{1}(z)=z$. In this section we assume $a_{i}(z) \in \mathbb{C}[[z]]$ $(1 \leq i \leq m)$. Let

$$
\varphi_{i}(z)=z\left(1+\sum_{j=p}^{\infty} b_{i, j} z^{j}\right), \quad i \geq 2,
$$

where $b_{i, p} \neq 0$ for some $i \geq 2$.

\subsection{Solutions of formal power series.}

Let $f(z)=\sum_{n=0}^{\infty} f_{n} z^{n} \in \mathbb{C}[[z]]$ and consider

$$
L(z, u(z))=f(z)
$$

First we have the existence of a solution of formal power series of (2.4). Let

$$
\mathscr{A}(z)=\sum_{i=1}^{m} a_{i}(z) .
$$

TheOREM 2.1. There exists a unique solution $u(z) \in \mathbb{C}[[z]]$ of $(2.4)$, if the following (1) or (2) holds.

(1) There exists $q \in \mathbb{Z}_{\geq 0}$ with $0 \leq q<p$ such that $\mathscr{A}(0)=\cdots=\mathscr{A}^{(q-1)}(0)=0$, $\mathscr{A}^{(q)}(0) \neq 0$ and $f_{0}=f_{1}=\cdots=f_{q-1}=0$.

(2) $\mathscr{A}(0)=\cdots=\mathscr{A}^{(p-1)}(0)=0,\left(\mathscr{A}^{(p)}(0) / p !\right)+n\left(\sum_{i=1}^{m} a_{i}(0) b_{i, p}\right) \neq 0$ for $n \in \mathbb{Z}_{\geq 0}$ and $f_{0}=f_{1}=\cdots=f_{p-1}=0$. 
TheOREM 2.2. Suppose $\mathscr{A}(0)=\cdots=\mathscr{A}^{(p)}(0)=0, \sum_{i=1}^{m} a_{i}(0) b_{i, p} \neq 0$ and $f_{0}=f_{1}=\cdots=f_{p}=0$. Then for given $c_{0}$ there is a unique solution $u(z) \in \mathbb{C}[[z]]$ of (2.4) with $u(0)=c_{0}$.

Let $u(z)=\sum_{n=0}^{\infty} c_{n} z^{n}$ be a formal solution of (2.4). Before proofs of Theorems 2.1 and 2.2 let us get relations of $\left\{c_{n}\right\}_{n \in \mathbb{Z}_{\geq 0}}$. Let $a_{i}(z)=\sum_{j=0}^{\infty} a_{i, j} z^{j}$ and define constants $\left\{B_{k, \ell}^{i} ; k, \ell \in \mathbb{Z}_{\geq 0}, 1 \leq i \leq m\right\}$ by

$$
\left(\varphi_{i}(z)\right)^{k}=z^{k}\left(1+\sum_{j=p}^{\infty} b_{i, j} z^{j}\right)^{k}=z^{k}\left(\sum_{\ell=0}^{\infty} B_{k, \ell}^{i} z^{\ell}\right),
$$

where

$$
B_{k, 0}^{i}=1, B_{k, \ell}^{i}=0 \quad(1 \leq \ell<p), B_{k, p}^{i}=k b_{i, p} .
$$

We have

$$
\begin{aligned}
a_{i}(z) u\left(\varphi_{i}(z)\right) & =\left(\sum_{j=0}^{\infty} a_{i, j} z^{j}\right)\left(\sum_{k=0}^{\infty} c_{k} z^{k}\left(\sum_{\ell=0}^{\infty} B_{k, \ell}^{i} z^{\ell}\right)\right) \\
& =\sum_{n=0}^{\infty}\left(\sum_{j+k+\ell=n} a_{i, j} c_{k} B_{k, \ell}^{i}\right) z^{n} .
\end{aligned}
$$

Let us consider the set

$$
\mathcal{N}_{n}=\left\{(j, k, \ell) \in \mathbb{Z}_{\geq 0}^{3} ; j+k+\ell=n, \ell=0, p, p+1, \cdots \cdots\right\} .
$$

More concretely

$$
\begin{aligned}
\mathcal{N}_{n}= & \{(0, n, 0)\} \cup\{(1, n-1,0)\} \cup \cdots \cup\{(p, n-p, 0)\} \cup\{(0, n-p, p)\} \\
& \cup\left\{(j, k, \ell) \in \mathcal{N}_{n} ; k<n-p\right\} .
\end{aligned}
$$

From (2.8) and (2.10)

$$
\begin{aligned}
& \left(\sum_{i=1}^{m} a_{i, 0}\right) c_{n}+\left(\sum_{i=1}^{m} a_{i, 1}\right) c_{n-1}+\cdots+\left(\sum_{i=1}^{m} a_{i, q}\right) c_{n-q}+\cdots \\
& +\left(\sum_{i=1}^{m} a_{i, p-1}\right) c_{n-p+1}+\left(\sum_{i=1}^{m} a_{i, p}+\sum_{i=1}^{m} a_{i, 0} B_{n-p, p}^{i}\right) c_{n-p}+\left(\sum_{\substack{1 \leq i \leq m \\
j+k+\ell=n \\
k<n-p}} a_{i, j} c_{k} B_{k, \ell}^{i}\right) \\
& =\sum_{s=0}^{p-1} \frac{\mathscr{A}^{(s)}(0)}{s !} c_{n-s}+\left(\frac{\mathscr{A}^{(p)}(0)}{p !}+(n-p)\left(\sum_{i=1}^{m} a_{i, 0} b_{i, p}\right)\right) c_{n-p} \\
& \quad+\left(\sum_{\substack{1 \leq i \leq m \\
j+k+\ell=n \\
k<n-p}} a_{i, j} c_{k} B_{k, \ell}^{i}\right)=f_{n} .
\end{aligned}
$$


Proof of Theorem 2.1. We have for case (1)

$$
\frac{\mathscr{A}^{(q)}(0)}{q !} c_{n-q}+\left(\sum_{\substack{1 \leq i \leq m \\ j+k+\ell=n \\ k<n-q}} a_{i, j} c_{k} B_{k, \ell}^{i}\right)=f_{n}
$$

and for case (2)

$$
\left(\frac{\mathscr{A}^{(p)}(0)}{p !}+(n-p)\left(\sum_{i=1}^{m} a_{i, 0} b_{i, p}\right)\right) c_{n-p}+\left(\sum_{\substack{1 \leq i \leq m \\ j+k+\ell=n \\ k<n-p}} a_{i, j} c_{k} B_{k, \ell}^{i}\right)=f_{n}
$$

The coefficients $\left\{c_{n}\right\}_{n \geq 0}$ are uniquely determined.

Proof of Theorem 2.2. We have

$$
(n-p)\left(\sum_{i=1}^{m} a_{i, 0} b_{i, p}\right) c_{n-p}+\left(\sum_{\substack{1 \leq i \leq m \\ j+k+\ell=n \\ k<n-p}} a_{i, j} c_{k} B_{k, \ell}^{i}\right)=f_{n} .
$$

$c_{0}$ is arbitrary and $c_{n-p}(n \geq p+1)$ are determined by (2.14).

As for the estimate of the coefficients of formal solutions in $\mathbb{C}[[z]]$ we have

TheOREm 2.3. Let $u(z) \in \mathbb{C}[[z]]$ be a formal solution of (2.4). Suppose that $a_{i}(z) \in \mathbb{C}_{\{1 / p\}}[[z]](1 \leq i \leq m)$ and $f(z) \in \mathbb{C}_{\{1 / p\}}[[z]]$, and the following (1) or (2) holds.

(1) $\mathscr{A}(0) \neq 0$.

(2) $\mathscr{A}(0)=\cdots=\mathscr{A}^{(p-1)}(0)=0$ and $\sum_{i=1}^{m} a_{i}(0) b_{i, p} \neq 0$.

Then $u(z) \in \mathbb{C}[[z]]$ belongs to $\mathbb{C}_{\{1 / p\}}[[z]]$.

We give a proof of Theorem 2.3 in the Section 5 .

REMARK 2.4. Let $u(z) \in \mathbb{C}[[z]]$ be a formal solution of (2.4). We may assume that $u(z)$ satisfies $u(0)=0$, by considering $u(z)-c_{0}$ as an unknown function. More generally, for a given $N$ let $v(z)=u(z)-\sum_{n=0}^{N-1} c_{n} z^{n}$. Then we may assume that $u(z)$ satisfies $u(z)=O\left(z^{N}\right)$, by considering $v(z)$ as an unknown function.

Suppose that (2) in Theorem 2.3 holds. Then there exists $n_{0} \in \mathbb{Z}_{\geq 0}$ such that $\left(\mathscr{A}^{(p)}(0) / p !\right)+n\left(\sum_{i=1}^{m} a_{i}(0) b_{i, p}\right) \neq 0$ for $n \geq n_{0}$. If $u(z) \in \mathbb{C}[[z]]$ with $u(z)=O\left(z^{n_{0}}\right)$ satisfies $L(z, u(z))=0$, then $u(z) \equiv 0$.

\subsection{Formal solutions of a homogeneous equation.}

In this subsection we construct solutions of a homogeneous equation

$$
\sum_{i=1}^{m} a_{i}(z) u\left(\varphi_{i}(z)\right)=0 .
$$


TheOREM 2.5. Suppose that $\mathscr{A}^{(i)}(0)=0$ for $0 \leq i \leq p-1$ and $\sum_{i=1}^{m} a_{i}(0) b_{i, p} \neq 0$. Then there exist a constant $\alpha$ and a formal solution $u(z)=z^{\alpha} w(z)$ of $(2.15)$, where $w(z) \in \mathbb{C}[[z]]$ with $w(0)=1$.

Proof. $\quad$ Set $u(z)=z^{\alpha} w(z)$ and let us find $\alpha$. From

$$
\begin{aligned}
u\left(\varphi_{i}(z)\right) & =\left(\varphi_{i}(z)\right)^{\alpha} w\left(\varphi_{i}(z)\right) \\
& =z^{\alpha}\left(1+b_{i, p} z^{p}+O\left(z^{p+1}\right)\right)^{\alpha} w\left(\varphi_{i}(z)\right) \\
& =z^{\alpha}\left(1+\alpha b_{i, p} z^{p}+O\left(z^{p+1}\right)\right) w\left(\varphi_{i}(z)\right)
\end{aligned}
$$

we have

$$
\sum_{i=1}^{m} a_{i}(z) u\left(\varphi_{i}(z)\right)=z^{\alpha} \sum_{i=1}^{m} g_{i}(z) w\left(\varphi_{i}(z)\right)
$$

where $g_{i}(z)=a_{i}(z)\left(1+\alpha b_{i, p} z^{p}+O\left(z^{p+1}\right)\right)$. Put $G(z)=\sum_{i=1}^{m} g_{i}(z)$. Then

$$
G^{(s)}(z)=\sum_{i=1}^{m}\left(\sum_{\ell=0}^{s}\left(\begin{array}{l}
s \\
\ell
\end{array}\right) \frac{d^{\ell}}{d z^{\ell}} a_{i}(z) \frac{d^{s-\ell}}{d z^{s-\ell}}\left(1+\alpha b_{i, p} z^{p}+O\left(z^{p+1}\right)\right)\right),
$$

$G^{(s)}(0)=0$ for $0 \leq s<p$ and

$$
G^{(p)}(0)=\alpha p !\left(\sum_{i=1}^{m} a_{i}(0) b_{i, p}\right)+\sum_{i=1}^{m} a_{i}^{(p)}(0) .
$$

Take $\alpha$ such that $G^{(p)}(0)=0$. Thus we get an equation $\sum_{i=1}^{m} g_{i}(z) w\left(\varphi_{i}(z)\right)=0$ with $G^{(s)}(0)=0$ for $0 \leq s \leq p$ and $\sum_{i=1}^{m} g_{i}(0) b_{i, p}=\sum_{i=1}^{m} a_{i}(0) b_{i, p} \neq 0$. It follows from Theorem 2.2 that there exists $w(z) \in \mathbb{C}[[z]]$ with $w(0)=1$ such that $\sum_{i=1}^{m} g_{i}(z) w\left(\varphi_{i}(z)\right)=0$ and $u(z)=z^{\alpha} w(z)$ is a desired formal solution of (2.15).

THEOREM 2.6. Suppose that there exists $\xi_{0}$ such that $\sum_{i=1}^{m} a_{i}(0) e^{-p b_{i, p} \xi_{0}}=0$ and $\sum_{i=1}^{m} a_{i}(0) b_{i, p} e^{-p b_{i, p} \xi_{0}} \neq 0$. Then there exists a formal solution of (2.15) in the form

$$
u(z)=\exp \left(\frac{C_{p}}{z^{p}}+\frac{C_{p-1}}{z^{p-1}}+\cdots+\frac{C_{1}}{z}\right) z^{\alpha} w(z),
$$

where $C_{p}=\xi_{0}$ and $w(z) \in \mathbb{C}[[z]]$ with $w(0)=1$.

Proof. Put

$$
u(z)=\exp \left(\frac{C_{p}}{z^{p}}+\frac{C_{p-1}}{z^{p-1}}+\cdots+\frac{C_{1}}{z}\right) v(z)
$$

and let us determine constants $\left\{C_{i}\right\}_{i=1}^{p}$. It follows from

$$
\varphi_{i}(z)=z\left(1+b_{i, p} z^{p}+O\left(z^{p+1}\right)\right)=\frac{z}{1-b_{i, p} z^{p}+O\left(z^{p+1}\right)}
$$


and

$$
\begin{aligned}
\frac{1}{\varphi_{i}(z)^{k}} & =\frac{1}{z^{k}}\left(1-b_{i, p} z^{p}+O\left(z^{p+1}\right)\right)^{k} \\
& =\frac{1}{z^{k}}-k b_{i, p} z^{p-k}+O\left(z^{p-k+1}\right)
\end{aligned}
$$

that

$$
\left\{\begin{aligned}
u\left(\varphi_{i}(z)\right) & =\exp \left(\sum_{k=1}^{p} \frac{C_{k}}{z^{k}}+\psi_{i}(z)\right) v\left(\varphi_{i}(z)\right), \\
\psi_{i}(z) & =-\sum_{k=1}^{p} C_{k}\left(k b_{i, p} z^{p-k}+O\left(z^{p-k+1}\right)\right), \\
\psi_{i}(0) & =-C_{p} p b_{i, p} .
\end{aligned}\right.
$$

Hence we have

$$
\sum_{i=1}^{m} a_{i}(z) u\left(\varphi_{i}(z)\right)=\sum_{i=1}^{m} a_{i}(z) \exp \left(\sum_{k=1}^{p} \frac{C_{k}}{z^{k}}+\psi_{i}(z)\right) v\left(\varphi_{i}(z)\right) .
$$

Thus we get the following functional equation

$$
\sum_{i=1}^{m} A_{i}(z) v\left(\varphi_{i}(z)\right)=0, \quad A_{i}(z)=a_{i}(z) e^{\psi_{i}(z)} .
$$

Set $G(z)=\sum_{i=1}^{m} A_{i}(z)$. We determine $\left\{C_{k}\right\}_{k=1}^{p}$ such that $G^{(s)}(0)=0$ for $0 \leq s \leq p-1$. By $G(0)=0$ we have

$$
\sum_{i=1}^{m} a_{i}(0) e^{-p b_{i, p} C_{p}}=0,
$$

which has a simple solution $C_{p}=\xi_{0}$. Then $\psi_{i}(0)=-p b_{i, p} \xi_{0}$ and we have

$$
\left\{\begin{array}{l}
G^{\prime}(z)=\sum_{i=1}^{m}\left(a_{i}^{\prime}(z) e^{\psi_{i}(z)}+a_{i}(z) e^{\psi_{i}(z)} \psi_{i}^{\prime}(z)\right), \\
G^{\prime}(0)=\sum_{i=1}^{m}\left(a_{i}^{\prime}(0) e^{\psi_{i}(0)}+a_{i}(0) e^{\psi_{i}(0)} \psi_{i}^{\prime}(0)\right), \\
\psi_{i}^{\prime}(0)=-C_{p-1}(p-1) b_{i, p}+C_{i}(p),
\end{array}\right.
$$

where $C_{i}(p)$ is a constant determined by $C_{p}$. Hence

$$
C_{p-1}=\frac{\sum_{i=1}^{m}\left(a_{i}^{\prime}(0)+a_{i}(0) C_{i}(p)\right) e^{-p b_{i, p} \xi_{0}}}{(p-1)\left(\sum_{i=1}^{m} a_{i}(0) b_{i, p} e^{-p b_{i, p} \xi_{0}}\right)} .
$$

Assume that $\left\{C_{k}\right\}_{p-s+1 \leq k \leq p}$ are determined. Then 


$$
\begin{aligned}
\psi_{i}(z)= & -\sum_{k=1}^{p} C_{k}\left(k b_{i, p} z^{p-k}+O\left(z^{p-k+1}\right)\right) \\
= & -\sum_{k=1}^{p-s} C_{k}\left(k b_{i, p} z^{p-k}+O\left(z^{p-k+1}\right)\right) \\
& + \text { a function determined by }\left\{C_{k}\right\}_{p-s+1 \leq k \leq p} .
\end{aligned}
$$

If $\ell \leq s-1, \psi_{i}^{(\ell)}(0)$ is a constant determined by $\left\{C_{k}\right\}_{p-s+1 \leq k \leq p}$. We have

$$
\begin{aligned}
G^{(s)}(z) & =\sum_{i=1}^{m} \sum_{\ell=0}^{s}\left(\begin{array}{l}
s \\
\ell
\end{array}\right)\left(a_{i}(z)\right)^{(s-\ell)}\left(e^{\psi_{i}(z)}\right)^{(\ell)} \\
& =\sum_{i=1}^{m} a_{i}(z)\left(e^{\psi_{i}(z)}\right)^{(s)}+\sum_{i=1}^{m} \sum_{\ell=0}^{s-1}\left(\begin{array}{l}
s \\
\ell
\end{array}\right)\left(a_{i}(z)\right)^{(s-\ell)}\left(e^{\psi_{i}(z)}\right)^{(\ell)}
\end{aligned}
$$

and

$$
\begin{aligned}
G^{(s)}(0)= & \sum_{i=1}^{m} a_{i}(0) e^{\psi_{i}(0)} \psi_{i}^{(s)}(0) \\
& + \text { a constant determined by }\left\{C_{k}\right\}_{k=p-s+1}^{p} . \\
= & -(p-s) s !\left(\sum_{i=1}^{m} a_{i}(0) b_{i, p} e^{-p b_{i, p} \xi_{0}}\right) C_{p-s} \\
& + \text { a constant determined by }\left\{C_{k}\right\}_{k=p-s+1}^{p} .
\end{aligned}
$$

We can take $C_{p-s}$ such that $G^{(s)}(0)=0$ and $\left\{C_{k}\right\}_{k=1}^{p}$ are successively determined. Thus we get

$$
\sum_{i=1}^{m} A_{i}(z) v\left(\varphi_{i}(z)\right)=0,
$$

where $G(z)=\sum_{i=1}^{m} A_{i}(z)$ satisfies

$$
\begin{aligned}
& G^{(s)}(0)=0 \quad 0 \leq s \leq p-1, \\
& \sum_{i=1}^{m} A_{i}(0) b_{i, p}=\sum_{i=1}^{m} a_{i}(0) b_{i, p} e^{-p b_{i, p} \xi_{0}} \neq 0 .
\end{aligned}
$$

It follows from Theorem 2.5 that there exists a formal solution $v(z)=z^{\alpha} w(z)$ of $(2.23)$ with $w(z) \in \mathbb{C}[[z]]$ and $w(0)=1$. Hence $u(z)=\exp \left(\sum_{s=1}^{p} C_{s} z^{-s}\right) z^{\alpha} w(z)$ is a desired formal solution of $(2.15)$.

\subsection{The similarity between solutions of the functional equations and irregular singular solutions of ordinary differential equations.}

The existence of solutions of formal power series are shown in Theorems 2.1, 2.2 and 2.3. Theorem 2.6 is the existence of formal solutions of the homogeneous equation, 
which are represented with exponential factors. These facts are similar to the properties of solutions of ordinary differential equations with an irregular singularity at $z=0$. Let

$$
P=z^{k} \frac{d}{d z}-A(z)
$$

be a system of ordinary differential operators, where $A(z)$ is an $n \times n$ matrix whose entries are holomorphic in $\{|z|<r\}$. Assume that $z=0$ is an irregular singular point of (2.25), necessarily $k \geq 2$. The following (a) and (b) are known.

(a) If $\boldsymbol{u} \in(\mathbb{C}[[z]])^{n}$ satisfies $P \boldsymbol{u} \in(\mathbb{C}\{z\})^{n}$, then there exists $\gamma>0$ determined by $P$ such that $\boldsymbol{u} \in\left(\mathbb{C}_{\{1 / \gamma\}}[[z]]\right)^{n}$.

(b) The homogeneous equation $P Y=0$ possesses a formal fundamental matrix solution

$$
Y(z)=\widehat{Y}(z) z^{L} e^{Q(z)}
$$

Here $Q(z)$ is a diagonal matrix whose entries are polynomials in $z^{-1 / \sigma}$ and not a constant matrix, $L$ is a constant matrix and the entries of $\hat{Y}$ belong to $\mathbb{C}\left[\left[z^{1 / \sigma}\right]\right]$, where $\sigma$ is a positive integer.

For the theory of ordinary differential equations about these topics we refer to Balser, Braaksma, Ramis and Sibuya $[\mathbf{3}]$ and Wasow $[\mathbf{6}]$. We notice that Theorem 2.3 corresponds to (a) and Theorem 2.6 corresponds to (b).

\section{Borel summability.}

In this section we give fundamental properties of Borel summability, Laplace transform and Borel transform. For the details of the topics in this section we refer to Balser $[\mathbf{1}],[\mathbf{2}]$. First we define a subset of $\mathbb{C}[[z]]$.

Definition 3.1. Let $\gamma>0$. We denote by $\mathbb{C}_{\gamma, \theta}\{z\}$ the set of all $w(z)=$ $\sum_{n=0}^{\infty} c_{n} z^{n} \in \mathbb{C}[[z]]$ such that there exist a sector $S(\theta, \delta, r)$ with $\delta>\pi / 2 \gamma$ and a holomorphic function $W(z)$ on $S(\theta, \delta, r)$ which satisfies

$$
\left|W(z)-\sum_{n=0}^{N-1} c_{n} z^{n}\right| \leq A C^{N} \Gamma\left(\frac{N}{\gamma}+1\right)|z|^{N}
$$

in $S(\theta, \delta, r)$ for all $N=1,2, \cdots$. We say that $w(z)$ is $\gamma$-Borel summable in a direction $\theta$.

From estimate (3.1) $\mathbb{C}_{\gamma, \theta}\{z\} \subset \mathbb{C}_{\{1 / \gamma\}}[[z]]$ holds. It follows from $\delta>\pi / 2 \gamma$ and (3.1) that $W(z)$ is uniquely determined for $w(z)$. So $W(z)$ is called the $\gamma$-Borel sum of $w(z)$ and we may identify $W(z)$ with $w(z)$. We denote (3.1) by

$$
W(z) \sim \sum_{n=0}^{\infty} c_{n} z^{n} .
$$

For our purposes we give notations of sectors in $\xi$-space. Put $S^{*}(\theta, \delta, R)=\{\xi ; 0<|\xi|<$ $R,|\arg \xi-\theta|<\delta\}, S^{*}(\theta, \delta)=\{\xi ;|\arg \xi-\theta|<\delta\}$ and $S_{\{0\}}^{*}(\theta, \delta)=\{\xi ;|\arg \xi-\theta|<$ 
$\rho(\arg \xi)\}$, where $\rho(\cdot)$ is some positive continuous function on $(\theta-\delta, \theta+\delta)$.

Definition 3.2. Let $\gamma>0$ and $S^{*}=S^{*}(\theta, \delta)$. The set of all $\phi(\xi) \in \mathcal{O}\left(S^{*}\right)$ with

$$
|\phi(\xi)| \leq A \exp \left(c|\xi|^{\gamma}\right) \quad \text { for } \xi \in S^{*} \cap\{|\xi| \geq 1\}
$$

for some $c>0$ is denoted by $\operatorname{Exp}\left(\gamma, S^{*}\right)$.

We define Laplace transform, Borel transform and convolution. Let $\phi(\xi) \in$ $\operatorname{Exp}\left(\gamma, S^{*}\right), S^{*}=S^{*}(\theta, \delta)$, and there exist $A, \epsilon>0$ such that

$$
|\phi(\xi)| \leq A|\xi|^{\epsilon-\gamma} \quad \text { for } \xi \in S^{*} \cap\{|\xi| \leq 1\} .
$$

Then the $\gamma$-Laplace transform $\mathcal{L}_{\gamma, \theta} \phi$ is defined by

$$
\left(\mathcal{L}_{\gamma, \theta} \phi\right)(z)=\int_{0}^{\infty e^{i \theta}} \exp \left(-\left(\frac{\xi}{z}\right)^{\gamma}\right) \phi(\xi) d \xi^{\gamma} \quad d \xi^{\gamma}=\gamma \xi^{\gamma-1} d \xi
$$

which is holomorphic in a region $S_{\{0\}}(\theta, \pi / 2 \gamma+\delta)$. Let $\psi(z)$ be holomorphic in $S_{\{0\}}(\theta, \pi / 2 \gamma+\delta)(0<\delta<\pi / 2 \gamma)$ with $|\psi(z)| \leq C|z|^{c}(c>0)$. Then the $\gamma$-Borel transform $\mathcal{B}_{\gamma, \theta} \psi$ is defined by

$$
\left(\mathcal{B}_{\gamma, \theta} \psi\right)(\xi)=\frac{1}{2 \pi i} \int_{\mathcal{C}} \exp \left(\frac{\xi}{z}\right)^{\gamma} \psi(z) d z^{-\gamma},
$$

where $\mathcal{C}$ is a path in $S_{\{0\}}(\theta, \pi / 2 \gamma+\delta)$ from $0 \exp \left(i\left(\theta+\pi / 2 \gamma+\delta^{\prime}\right)\right)$ to $0 \exp \left(i\left(\theta-\pi / 2 \gamma-\delta^{\prime}\right)\right)$ $\left(0<\delta^{\prime}<\delta\right)$. We denote $\left(\mathcal{B}_{\gamma, \theta} \psi\right)(\xi)$ simply by $\widehat{\psi}(\xi)$ which belongs to $\operatorname{Exp}\left(\gamma, S^{*}\left(\theta, \delta_{0}\right)\right)$ for any $\delta_{0}$ with $0<\delta_{0}<\delta^{\prime}$.

Let $w_{0}(\xi)$ be a holomorphic function on $\Omega=\{0<|\xi|<R\} \cup S^{*}, S^{*}=S^{*}(\theta, \delta)$, such that

$$
\left\{\begin{array}{l}
w_{0}(\xi)=\sum_{n=1}^{\infty} \widehat{c}_{n} \xi^{n-\gamma} \quad \text { in }\{0<|\xi|<R\} \\
\left|w_{0}(\xi)\right| \leq A \exp \left(c|\xi|^{\gamma}\right) \quad \text { in } S^{*} \cap\{|\xi| \geq 1\}
\end{array}\right.
$$

Then

$$
W_{0}(z)=\int_{0}^{\infty e^{i \theta}} \exp \left(-\left(\frac{\xi}{z}\right)^{\gamma}\right) w_{0}(\xi) d \xi^{\gamma}
$$

is $\gamma$-Borel summable in a direction $\theta$ such that $w_{0}(\xi)=\widehat{W}_{0}(\xi)$ and

$$
W_{0}(z) \sim \sum_{n=1}^{\infty} \widehat{c}_{n} \Gamma(n / \gamma) z^{n}
$$

Let $\phi_{i}(\xi) \in \mathcal{O}\left(S_{\{0\}}^{*}(\theta, \delta)\right)(i=1,2)$ with $\left|\phi_{i}(\xi)\right| \leq C|\xi|^{\epsilon-\gamma}$. We define the $\gamma$ convolution of $\phi_{1}(\xi)$ and $\phi_{2}(\xi)$ by 


$$
\left(\phi_{1} \underset{\gamma}{*} \phi_{2}\right)(\xi)=\int_{0}^{\xi} \phi_{1}\left(\left(\xi^{\gamma}-\eta^{\gamma}\right)^{1 / \gamma}\right) \phi_{2}(\eta) d \eta^{\gamma} \quad \xi \in S_{\{0\}}^{*}(\theta, \delta) .
$$

The followings hold.

Proposition 3.3. Let $S^{*}=S^{*}(\theta, \delta)$ and $\phi_{i}(\xi) \in \operatorname{Exp}\left(\gamma, S^{*}\right)$ with $\left|\phi_{i}(\xi)\right| \leq$ $C|\xi|^{\epsilon-\gamma}(\epsilon>0)(i=0,1,2)$ on $\left\{\xi \in S^{*} ; 0<|\xi| \leq 1\right\}$. Then

$$
\begin{aligned}
\mathcal{B}_{\gamma, \theta} \mathcal{L}_{\gamma, \theta} \phi_{0} & =\phi_{0}, \\
\left(\mathcal{L}_{\gamma, \theta} \phi_{1}\right)\left(\mathcal{L}_{\gamma, \theta} \phi_{2}\right) & =\mathcal{L}_{\gamma, \theta}\left(\phi_{1} \underset{\gamma}{*} \phi_{2}\right) .
\end{aligned}
$$

The following Lemma is given in Ōuchi [4], [5].

Lemma 3.4. Let $0<\gamma \leq \kappa$. Let $\phi_{i}(\xi) \in \mathcal{O}\left(S_{\{0\}}(\theta, \delta)\right)(i=1,2)$ with

$$
\left|\phi_{i}(\xi)\right| \leq \frac{C_{i}|\xi|^{s_{i}-\gamma} e^{c|\xi|^{\kappa}}}{\Gamma\left(s_{i} / \gamma\right)},
$$

where $s_{1}, s_{2}>0, c \geq 0$. Then $\left(\phi_{1} \underset{\gamma}{*} \phi_{2}\right)(\xi) \in \mathcal{O}\left(S_{\{0\}}(\theta, \delta)\right)$ and

$$
\left|\left(\phi_{1} \underset{\gamma}{*} \phi_{2}\right)(\xi)\right| \leq \frac{C_{1} C_{2}|\xi|^{s_{1}+s_{2}-\gamma} e^{c|\xi|^{\kappa}}}{\Gamma\left(\left(s_{1}+s_{2}\right) / \gamma\right)} .
$$

\section{Integral representation of solutions.}

The aim of this section is to show that formal solutions are Borel summable. For our aim we introduce a condition. Let $b_{1, p}=0$ and $B=\left\{b_{i, p} ; 1 \leq i \leq m\right\} \subset \mathbb{C}$.

Condition B. $a_{1}(0) \neq 0, b_{i, p} \neq 0$ for $2 \leq i \leq m$ and $\{0\}$ is a vertex of the convex hull $\widehat{B}$ of $B$.

First we give a lemma that follows from Condition B.

Lemma 4.1. Assume Condition B. Let

$$
h(\xi)=\sum_{i=1}^{m} a_{i, 0} e^{p b_{i, p} \xi^{p}}, \quad a_{i, 0}=a_{i}(0) .
$$

Then there exist a sector $S^{*}\left(\theta_{0}, \varepsilon_{0}\right)$ and positive constants $r_{0}, d$ and $M$ such that

(1) $\left|e^{p b_{i, p} \xi^{p}}\right| \leq e^{-d|\xi|^{p}}(i \geq 2)$ on $S^{*}\left(\theta_{0}, \varepsilon_{0}\right)$,

(2) $h(\xi) \neq 0$ on $S^{*}\left(\theta_{0}, \varepsilon_{0}\right) \cup\left\{0<|\xi|<r_{0}\right\}$, and if $h(0) \neq 0,|h(\xi)|^{-1} \leq M$ holds on $S^{*}\left(\theta_{0}, \varepsilon_{0}\right) \cup\left\{|\xi|<r_{0}\right\}$.

We refer the proof of Lemma 4.1 to [5]. In the following of this section we assume Condition B. Let $\theta_{0}$ and $S^{*}=S^{*}\left(\theta_{0}, \varepsilon_{0}\right)$ be those in Lemma 4.1 and $\varepsilon_{0}>0$ be so small, if necessary. Let us recall $\mathscr{A}(z)=\sum_{i=1}^{m} a_{i}(z)$.

Theorem 4.2. Assume Condition B and suppose the following (1) or (2) holds. 
(1) $\mathscr{A}(0) \neq 0$,

(2) $\mathscr{A}(0)=\cdots=\mathscr{A}^{(p-1)}(0)=0, \quad \sum_{i=1}^{m} a_{i}(0) b_{i, p} \neq 0$.

Then any formal solution $u(z) \in \mathbb{C}[[z]]$ of $(2.4)$ is $p$-Borel summable in the direction $\theta_{0}$, provided $a_{i}(z)(1 \leq i \leq m)$ and $f(z)$ are $p$-Borel summable in the direction $\theta_{0}$

Corollary 4.3. Assume Condition B. Let $w(z) \in \mathbb{C}[[z]]$ be that in Theorem 2.5 or Theorem 2.6. Then $w(z)$ is $p$-Borel summable in the direction $\theta_{0}$, provided $a_{i}(z)(1 \leq$ $i \leq m$ ) are $p$-Borel summable in the direction $\theta_{0}$.

Since the equation that determines $w(z) \in \mathbb{C}[[z]]$ in Theorem 2.5 or Theorem 2.6 satisfies the condition (2) in Theorem 4.2, Corollary 4.3 follows from Theorem 4.2.

In the following of this section we assume $\left\{a_{i}(z)\right\}_{i=1}^{m}$ and $f(z)$ are $p$-Borel summable in the direction $\theta_{0}$. We may assume $f(0)=0$ and a formal solution $u(z)$ of $(2.4)$ satisfies $u(0)=0$. In order to show Theorem 4.2 we try to represent $u(z)$ in the form

$$
u(z)=\int_{0}^{\infty e^{i \theta_{0}}} \exp \left(-\left(\frac{\xi}{z}\right)^{p}\right) \widehat{u}(\xi) d \xi^{p}, \quad d \xi^{p}=p \xi^{p-1} d \xi .
$$

Let us proceed to obtain an equation that $\widehat{u}(\xi)$ satisfies. The following procedure is similar to that in $[\mathbf{5}]$. By $\varphi_{i}(z)=z\left(1+b_{i, p} z^{p}+O\left(z^{p+1}\right)\right), \psi_{i}(z)$ is determined by

$$
\varphi_{i}(z)^{-p}=\frac{1-p b_{i, p} z^{p}-z^{p} \psi_{i}(z)}{z^{p}}, \quad \psi_{i}(z)=O(z) .
$$

We have

$$
\begin{aligned}
u\left(\varphi_{i}(z)\right) & =\int_{0}^{\infty e^{i \theta_{0}}} \exp \left(-\frac{\xi^{p}}{z^{p}}+p b_{i, p} \xi^{p}+\psi_{i}(z) \xi^{p}\right) \widehat{u}(\xi) d \xi^{p} \\
& =\sum_{\ell=0}^{\infty} \psi_{i}(z)^{\ell} \int_{0}^{\infty e^{i \theta_{0}}} \exp \left(-\frac{\xi^{p}}{z^{p}}+p b_{i, p} \xi^{p}\right) \frac{\xi^{p \ell}}{\ell !} \widehat{u}(\xi) d \xi^{p}
\end{aligned}
$$

and

$$
\begin{aligned}
& \sum_{i=1}^{m} a_{i}(z) u\left(\varphi_{i}(z)\right) \\
& =\sum_{i=1}^{m} \sum_{\ell=0}^{\infty} a_{i}(z) \psi_{i}(z)^{\ell} \int_{0}^{\infty e^{i \theta_{0}}} \exp \left(-\frac{\xi^{p}}{z^{p}}\right)\left(\frac{\xi^{p \ell} e^{p b_{i, p} \xi^{p}}}{\ell !} \widehat{u}(\xi)\right) d \xi^{p}
\end{aligned}
$$

Let $a_{i}(z)=a_{i, 0}+a_{i}^{\prime}(z)$, where $a_{i, 0}=a_{i}(0)$ and $a_{i}^{\prime}(z)=O(z)$. By using the $p$-Borel transforms of $a_{i}^{\prime}(z)$ and $\psi_{i}(z)$, 


$$
\begin{aligned}
& \sum_{i=1}^{m} a_{i}(z) u\left(\varphi_{i}(z)\right) \\
& =\sum_{i=1}^{m} \sum_{\ell=0}^{\infty} a_{i, 0} \int_{0}^{\infty e^{i \theta_{0}}} \exp \left(-\frac{\xi^{p}}{z^{p}}\right)(\overbrace{\widehat{\psi}_{i}^{*} \cdots \underset{p}{*} \widehat{\psi}_{i}}^{\ell}\left(\frac{\xi^{p \ell} e^{p b_{i, p} \xi^{p}}}{\ell !} \hat{u}(\xi)\right)) d \xi^{p} \\
& +\sum_{i=1}^{m} \sum_{\ell=0}^{\infty} \int_{0}^{\infty e^{i \theta_{0}}} \exp \left(-\frac{\xi^{p}}{z^{p}}\right)\left(\widehat{a}_{i}^{\prime} \underset{p}{* \widehat{\psi}_{i}^{*} \cdots \underset{p}{*} \hat{\psi}_{i} *}{ }_{p}^{\ell}\left(\frac{\xi^{p \ell} e^{p b_{i, p} \xi^{p}}}{\ell !} \widehat{u}(\xi)\right)\right) d \xi^{p} .
\end{aligned}
$$

Put

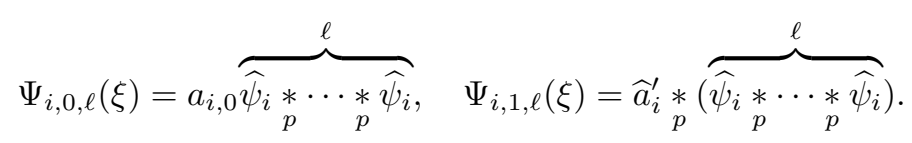

LEMMA 4.4. There exist a punctured disk $\{0<|\xi|<r\}$ and a sector $S^{*}\left(\theta_{0}, \delta\right)$ such that in $\Omega=\{0<|\xi|<r\} \cup S^{*}\left(\theta_{0}, \delta\right)$

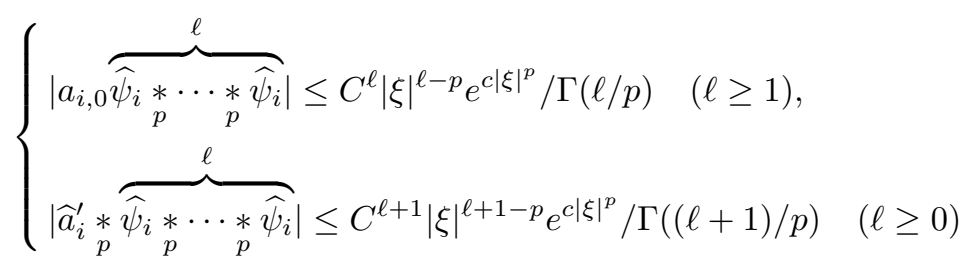

holds for some positive constants $C$ and $c$.

Proof. Since $\psi_{i}(z)$ and $a_{i}^{\prime}(z)$ are $p$-Borel summable in the direction $\theta_{0}$ and $\psi_{i}(0)=a_{i}^{\prime}(0)=0$, there exist a domain $\Omega$ and positive constants $C_{1}$ and $c$ such that $\left|\widehat{\psi}_{i}(\xi)\right|,\left|\widehat{a}_{i}^{\prime}(\xi)\right| \leq C_{1}|\xi|^{1-p} e^{c|\xi|^{p}}$ in $\Omega$. The estimate (4.5) follows from Lemma 3.4.

Define operators $\mathscr{P}_{\ell}$ and $\mathscr{Q}_{\ell}$ by

$$
\left\{\begin{array}{l}
\mathscr{P}_{\ell} v=\sum_{i=1}^{m} \Psi_{i, 0, \ell}^{*}\left(\frac{\xi_{p}^{p \ell} e^{p p_{p}^{i} \xi^{p}}}{\ell !} v(\xi)\right) \\
\mathscr{Q}_{\ell} v=\sum_{i=1}^{m} \Psi_{i, 1, \ell}{ }_{p}^{*}\left(\frac{\xi^{p \ell} e^{p b_{p}^{i} \xi^{p}}}{\ell !} v(\xi)\right)
\end{array}\right.
$$

Then we have formally

$$
\begin{aligned}
& \sum_{i=1}^{m} a_{i}(z) u\left(\varphi_{i}(z)\right) \\
= & \int_{0}^{\infty e^{i \theta_{0}}} \exp \left(-\left(\frac{\xi}{z}\right)^{p}\right)\left(h(\xi)+\sum_{\ell=1}^{\infty} \mathscr{P}_{\ell}+\sum_{\ell=0}^{\infty} \mathscr{Q}_{\ell}\right) \hat{u}(\xi) d \xi^{p} .
\end{aligned}
$$

Since $f(z)$ is $p$-Borel summable in the direction $\theta_{0}$, 


$$
f(z)=\int_{0}^{\infty e^{i \theta_{0}}} \exp \left(-\left(\frac{\xi}{z}\right)^{p}\right) \hat{f}(\xi) d \xi^{p} .
$$

Thus from $\sum_{i=1}^{m} a_{i}(z) u\left(\varphi_{i}(z)\right)=f(z)$ we obtain a convolution equation that $\widehat{u}$ satisfies, by denoting $\widehat{u}$ by $v$,

$$
h(\xi) v+\sum_{\ell=1}^{\infty} \mathscr{P}_{\ell} v+\sum_{\ell=0}^{\infty} \mathscr{Q}_{\ell} v=\widehat{f} .
$$

In case $\left\{a_{i}(z)\right\}_{1 \leq i \leq m}$ are constants, the terms $\mathscr{Q}_{\ell} v(\ell=0,1, \cdots)$ do not appear in [5].

Let us construct a solution of (4.9). There are 2 cases. The first one is $\mathscr{A}(0) \neq 0$, that is, $h(0) \neq 0$. We can construct a solution in the almost same way as the case $\sum_{i=1}^{m} a_{i} \neq 0$ in [5]. A solution $v(\xi)=\sum_{n=0}^{\infty} v_{n}(\xi)$ is constructed as follows.

$$
\left\{\begin{array}{l}
h(\xi) v_{0}(\xi)=\widehat{f}(\xi), \\
h(\xi) v_{n}(\xi)+\sum_{\ell=1}^{n} \mathscr{P}_{\ell} v_{n-\ell}+\sum_{\ell=0}^{n-1} \mathscr{Q}_{\ell} v_{n-\ell-1}=0
\end{array}\right.
$$

Dividing by $h(\xi)$, we obtain $v_{n}(\xi)$. By using Lemmas 4.1 and 4.4 and applying the method used for the case $\sum_{i=1}^{m} a_{i} \neq 0$ in [5] to this case, we obtain an estimate of $v_{n}(\xi)$, a representation in a punctured disk and the convergence of $v(\xi)=\sum_{n=0}^{\infty} v_{n}(\xi)$, which is a solution of (4.9). Hence we have the following Proposition and Theorem.

Proposition 4.5. Assume $\mathscr{A}(0) \neq 0$ and Condition B. Then there exist a sector $S^{*}\left(\theta_{0}, \varepsilon_{0}\right)$, a punctured disk $\left\{0<|\xi|<r_{0}\right\}$ and a unique solution $v_{n}(\xi) \in \mathcal{O}(\Omega), \Omega=$ $S^{*}\left(\theta_{0}, \varepsilon_{0}\right) \cup\left\{0<|\xi|<r_{0}\right\}$, of (4.10) such that

$$
\left|v_{n}(\xi)\right| \leq A C^{n}|\xi|^{n+1-p} e^{c|\xi|^{p}} / \Gamma((n+1) / p)
$$

for some positive constants $A, C$ and $c$, and

$$
v_{n}(\xi)=\sum_{j=n+1}^{\infty} v_{n, j}^{*} \xi^{j-p}
$$

in $\left\{0<|\xi|<r_{0}\right\}$.

TheOrem 4.6. Assume $\mathscr{A}(0) \neq 0$ and Condition B. Then there exist a sector $S^{*}\left(\theta_{0}, \varepsilon_{0}\right)$, a punctured disk $\left\{0<|\xi|<r_{0}\right\}$ and a unique solution $v(\xi) \in \mathcal{O}(\Omega)$ of (4.9), $\left.\Omega=S^{*}\left(\theta_{0}, \varepsilon_{0}\right) \cup\left\{0<|\xi|<r_{0}\right\}\right)$, such that

$$
|v(\xi)| \leq A|\xi|^{1-p} e^{c_{0}|\xi|^{p}} \quad \xi \in \Omega
$$

and

$$
v(\xi)=\sum_{j=1}^{\infty} v_{j}^{*} \xi^{j-p} \quad\left\{0<|\xi|<r_{0}\right\} .
$$


By Theorem 4.6 there exists a solution $v(\xi)$ of (4.9) with (4.13) and (4.14). Define

$$
U(z)=\int_{0}^{\infty e^{i \theta_{0}}} \exp \left(-(\xi / z)^{p}\right) v(\xi) d \xi^{p} .
$$

Then it is a solution of (2.4) that is $p$-Borel summable in the direction $\theta_{0}$ and its asymptotic expansion coincides with the unique formal power series solution. Hence Theorem 4.2 holds under the condition (1).

Next we study another case

$$
\mathscr{A}(0)=\mathscr{A}^{\prime}(0)=\cdots=\mathscr{A}^{(p-1)}(0)=0, \quad \sum_{i=1}^{m} a_{i}(0) b_{i, p} \neq 0 .
$$

The condition $\sum_{i=1}^{m} a_{i}(0) b_{i, p} \neq 0$ means that $\xi=0$ is a zero of $h(\xi)$ with order $p$ and there exists $n_{0} \in \mathbb{Z}_{\geq 0}$ such that

$$
n+\Re \mathscr{A}^{(p)}(0) /\left(p ! \sum_{i=1}^{m} a_{i}(0) b_{i, p}\right)>0 \text { for } n \geq n_{0} .
$$

In this case we construct a solution of (4.9) locally and show that it is holomorphically extensible to a sector $S^{*}\left(\theta_{0} \cdot \varepsilon_{0}\right)$. The method of construction of a local solution of $(4.9)$ is different from that in $[5]$. We note

$$
a_{i}(z)=\sum_{j=0}^{p} a_{i, j} z^{j}+a_{i}^{\prime \prime}(z), a_{i}^{\prime \prime}(z)=O\left(z^{p+1}\right),
$$

$\sum_{i=1}^{m} a_{i, j}=0$ for $0 \leq j \leq p-1$ and

$$
h(\xi)=p \xi^{p}\left(\left(\sum_{i=1}^{m} a_{i, 0} b_{i, p}\right)+h_{1}(\xi)\right), h_{1}(\xi)=O\left(\xi^{p}\right) .
$$

We have

$$
\begin{aligned}
& h(\xi) w+\mathscr{Q}_{0} w \\
= & p \xi^{p}\left(\left(\sum_{i=1}^{m} a_{i, 0} b_{i, p}\right)+h_{1}(\xi)\right) w(\xi)+\sum_{j=1}^{p} \sum_{i=1}^{m} a_{i, j} \frac{\xi^{j-p}}{\Gamma(j / p)} *\left(e^{p b_{i, p} \xi^{p}} w(\xi)\right) \\
& +\sum_{i=1}^{m} \hat{a}_{i}^{\prime \prime} \underset{p}{*}\left(e^{p b_{p}^{i} \xi^{p}} w(\xi)\right) \\
= & p \xi^{p}\left(\left(\sum_{i=1}^{m} a_{i, 0} b_{i, p}\right)+h_{1}(\xi)\right) w(\xi)+\left(\sum_{i=1}^{m} a_{i, p}\right) \underset{p}{*} w(\xi) \\
& +\sum_{j=1}^{p} \sum_{i=1}^{m} a_{i, j} \frac{\xi^{j-p}}{\Gamma(j / p)} \underset{p}{*}\left(\left(e^{p b_{i, p} \xi^{p}}-1\right) w(\xi)\right)+\sum_{i=1}^{m} \hat{a}_{i}^{\prime \prime} \underset{p}{*}\left(e^{p b_{i, p} \xi^{p}} w(\xi)\right) .
\end{aligned}
$$

Put 


$$
\begin{aligned}
& \mathscr{Q}_{*} w=p \xi^{p}\left(\left(\sum_{i=1}^{m} a_{i, 0} b_{i, p}\right)+h_{1}(\xi)\right) w(\xi)+\left(\sum_{i=1}^{m} a_{i, p}\right) \underset{p}{*} w(\xi), \\
& \mathscr{Q}_{0}^{\prime} w=\sum_{j=1}^{p} \sum_{i=1}^{m} a_{i, j} \frac{\xi^{j-p}}{\Gamma(j / p)} \underset{p}{*}\left(\left(e^{p b_{i, p} \xi^{p}}-1\right) w\right)+\sum_{i=1}^{m} \hat{a}_{i}^{\prime \prime} \underset{p}{*}\left(e^{p b_{i, p} \xi^{p}} w\right) .
\end{aligned}
$$

Then

$$
h(\xi)+\sum_{\ell=1}^{\infty} \mathscr{P}_{\ell}+\sum_{\ell=0}^{\infty} \mathscr{Q}_{\ell}=\mathscr{Q}_{*}+\mathscr{Q}_{0}^{\prime}+\sum_{\ell=1}^{\infty} \mathscr{P}_{\ell}+\sum_{\ell=1}^{\infty} \mathscr{Q}_{\ell}
$$

Lemma 4.7. Let $w(\xi) \in \mathcal{O}\{0<|\xi|<r\}$ with $|w(\xi)| \leq A|\xi|^{s-p} / \Gamma(s / p)(s>0)$. Then there is a constant $C$ independent of $w(\xi)$ such that

$$
\left|\mathscr{Q}_{0}^{\prime} w\right| \leq A C|\xi|^{s+1} / \Gamma((s+1) / p)
$$

Proof. We have estimates $|\xi|^{j-p} / \Gamma(j / p) \leq C_{1}|\xi|^{1-p} / \Gamma(1 / p)$ for $j \geq 1, \mid\left(e^{p b_{p}^{i} \xi^{p}}-\right.$ 1) $\left.w(\xi)\left|\leq A C_{1} s\right| \xi\right|^{s} / \Gamma(s / p+1)$ and $\left|\widehat{a}_{i}^{\prime \prime}(\xi)\right| \leq A C_{2}|\xi| / \Gamma(1 / p+1)$ in $\{0<|\xi| \leq r\}$. Hence

$$
\begin{aligned}
& \left|\frac{\xi^{j-p}}{\Gamma(j / p)} \underset{p}{*}\left(\left(e^{p b_{p}^{i} \xi^{p}}-1\right) w(\xi)\right)\right| \leq \frac{A C_{3} s|\xi|^{s+1}}{\Gamma((s+1) / p+1)} \leq \frac{A C_{4}|\xi|^{s+1}}{\Gamma((s+1) / p)}, \\
& \left|\widehat{a}_{i}^{\prime \prime} \underset{p}{*}\left(e^{p b_{p}^{i} \xi^{p}} w(\xi)\right)\right| \leq \frac{A C_{5}|\xi|^{s+1}}{\Gamma((s+1) / p+1)} \leq \frac{A C_{4}|\xi|^{s+1}}{\Gamma((s+1) / p)}
\end{aligned}
$$

and we have (4.21).

\section{Lemma 4.8. Consider}

$$
p \xi^{p}\left(\sum_{i=1}^{m} a_{i, 0} b_{i, p}+h_{1}(\xi)\right) w+\left(\sum_{i=1}^{m} a_{i, p} \underset{p}{*} w\right)=g(\xi),
$$

where $\sum_{i=1}^{m} a_{i, 0} b_{i, p}+h_{1}(\xi) \neq 0$ in $\{|\xi|<R\}$. Suppose $g(\xi)=\sum_{j=n_{1}}^{\infty} g_{j} \xi^{j}$ is holomorphic in $\{|\xi|<r\} \quad(r<R)$ with $|g(\xi)| \leq A|\xi|^{n_{1}}$, where $n_{1} \geq n_{0}$. Then there exist a unique holomorphic solution $w(\xi)=\sum_{j=n_{1}}^{\infty} c_{j} \xi^{j-p}$ in $\{0<|\xi|<r\}$ and a constant $C>0$ independent of $w(\xi)$ such that $|w(\xi)| \leq A C|\xi|^{n_{1}-p}$.

Proof. $\quad$ Let $v(\xi)=(1 \underset{p}{*} w)(\xi)$. Then $(d / d \xi) v(\xi)=p \xi^{p-1} w(\xi)$ and by $\sum_{i=1}^{m} a_{i, p}=$ $\mathscr{A}^{p}(0) / p$ ! the equation $(4.22)$ becomes

$$
\xi v^{\prime}(\xi)+A(\xi) v(\xi)=G(\xi),
$$

where 


$$
\left\{\begin{aligned}
A(\xi) & =\mathscr{A}^{(p)}(0) / p !\left(\sum_{i=1}^{m} a_{i, 0} b_{i, p}+h_{1}(\xi)\right) \\
& =A_{0}+\xi B(\xi), \quad A_{0}=\mathscr{A}^{(p)}(0) / p !\left(\sum_{i=1}^{m} a_{i, 0} b_{i, p}\right), \\
G(\xi) & =g(\xi) /\left(\sum_{i=1}^{m} a_{i, 0} b_{i, p}+h_{1}(\xi)\right) .
\end{aligned}\right.
$$

There exists a holomorphic solution of (4.23) such that $v(\xi)=\sum_{n=n_{1}}^{\infty} v_{n}^{\prime} \xi^{n}$ and

$$
v(\xi)=\xi^{-A_{0}} \int_{0}^{\xi} \exp \left(-\int_{\tau}^{\xi} B(s) d s\right) \tau^{A_{0}-1} G(\tau) d \tau .
$$

For $\alpha<\arg \xi<\beta$, by putting $A_{0}^{*}=\Re A_{0}$,

$$
|v(\xi)| \leq A C_{0}|\xi|^{-A_{0}^{*}} \int_{0}^{|\xi|} r^{n_{1}+A_{0}^{*}-1} d r \leq \frac{A C_{0}|\xi|^{n_{1}}}{n_{1}+A_{0}^{*}}
$$

and there is a constant $C_{1}$ such that

$$
\left|\xi v^{\prime}(\xi)\right| \leq|A(\xi) v(\xi)|+|G(\xi)| \leq A C_{1}|\xi|^{n_{1}} .
$$

Since $w(\xi)$ is single valued holomorphic in $\{0<|\xi|<r\}$, there is a constant $C>0$ independent of $w(\xi)$ such that

$$
|w(\xi)| \leq\left|\xi^{1-p} v^{\prime}(\xi) / p\right| \leq A C|\xi|^{n_{1}-p} .
$$

We construct a solution $v(\xi)=\sum_{n=0}^{\infty} v_{n}(\xi)$ of (4.9) in $\{0<|\xi|<r\}$ under the condition (4.16) as follows.

$$
\left\{\begin{array}{l}
\mathscr{Q}_{*} v_{0}=\widehat{f}(\xi), \\
\mathscr{Q}_{*} v_{1}+\mathscr{Q}_{0}^{\prime} v_{0}+\mathscr{P}_{1} v_{0}=0, \\
\mathscr{Q}_{*} v_{n}+\mathscr{Q}_{0}^{\prime} v_{n-1}+\sum_{\ell=1}^{n} \mathscr{P}_{\ell} v_{n-\ell}+\sum_{\ell=1}^{n-1} \mathscr{Q}_{\ell} v_{n-\ell-1}=0, \quad n \geq 2 .
\end{array}\right.
$$

Proposition 4.9. Suppose (4.16) holds. Let $\widehat{f}(\xi) \in \mathcal{O}(\{|\xi|<R\})$ with $\widehat{f}(\xi)=$ $\sum_{j=n_{0}+p+1}^{\infty} f_{j} \xi^{j-p}$. Then there exist $r>0$ and $v_{n}(\xi) \in \mathcal{O}(\{0<|\xi|<r\})\left(n \in \mathbb{Z}_{\geq 0}\right)$ satisfying (4.29) such that the following (1) and (2) hold.

(1) There exist positive constants $A$ and $C$ such that

$$
\left|v_{n}(\xi)\right| \leq \frac{A C^{n}|\xi|^{n+n_{0}+1-p}}{\Gamma\left(\left(n+n_{0}+1\right) / p\right)}
$$

and 


$$
v_{n}(\xi)=\sum_{j=n+n_{0}+1}^{\infty} v_{n, j} \xi^{j-p}
$$

(2) $v(\xi)=\sum_{n=0}^{\infty} v_{n}(\xi)$ converges in $\{0<|\xi|<r\}$. It takes the form of $v(\xi)=$ $\sum_{j=n_{0}+1}^{\infty} v_{j}^{*} \xi^{j-p}$ and is a solution of $(4.9)$ in $\{0<|\xi|<r\}$.

Proof. $\quad$ Since $\mathscr{Q}^{*} v_{0}(\xi)=\widehat{f}(\xi)$ and $|\widehat{f}(\xi)| \leq C_{1}|\xi|^{n_{0}+1}$, (4.30) and (4.31) hold for $n=0$ by Lemma 4.8. We assume (4.30) and (4.31) hold for $0 \leq n \leq N-1$. Then by Lemma 4.7

$$
\left|\mathscr{Q}_{0}^{\prime} v_{N-1}\right| \leq \frac{A C_{1} C^{N-1}|\xi|^{N+n_{0}+1}}{\Gamma\left(\left(N+n_{0}+1\right) / p\right)}
$$

We have

$$
\left|\frac{e^{p b_{i, p} \xi^{p \ell}}}{\ell !} v_{N-\ell}\right| \leq \frac{A C^{N-\ell} C_{0}^{\ell}|\xi|^{N-\ell+n_{0}+1}}{\Gamma\left(\left(N-\ell+n_{0}+1\right) / p\right) \ell !}
$$

for $\ell \geq 1$ and

$$
\left\{\begin{array}{l}
\left|\mathscr{P}_{\ell} v_{N-\ell}\right| \leq \frac{A C_{1}^{\ell} C^{N-\ell}|\xi|^{N+n_{0}+1}}{\Gamma\left(\left(N+n_{0}+1\right) / p\right) \ell !}, \\
\left|\mathscr{Q}_{\ell} v_{N-\ell-1}\right| \leq \frac{A C_{1}^{\ell+1} C^{N-\ell-1}|\xi|^{N+n_{0}+1}}{\Gamma\left(\left(N+n_{0}+1\right) / p\right) \ell !},
\end{array}\right.
$$

by using Lemmas 3.4 and 4.4. Hence for a large $C>0$

$$
\left|\mathscr{Q}^{*} v_{N}\right| \leq \frac{A C_{2} C^{N-1}|\xi|^{N+n_{0}+1}}{\Gamma\left(\left(N+n_{0}+1\right) / p\right)} .
$$

From Lemma $4.8\left|v_{N}(\xi)\right| \leq \frac{A C^{N}|\xi|^{N+n_{0}+1-p}}{\Gamma\left(\left(N+n_{0}+1\right) / p\right)}$ with

$$
v_{N}(\xi)=\sum_{j=N+n_{0}+1}^{\infty} v_{n, j} \xi^{j-p}
$$

in $\{0<|\xi|<r\}$. It follows from (4.30) and (4.31) that $v(\xi)=\sum_{n=0}^{\infty} v_{n}(\xi)$ converges and $v(\xi)=\sum_{j=n_{0}+1}^{\infty} v_{j}^{*} \xi^{j-p}$, which is a solution of (4.9).

We have constructed a solution $v(\xi)$ of $(4.9)$ in $\{0<|\xi|<r\}$ under the condition (4.16). As for the holomorphic extension of $v(\xi)$ we have

Theorem 4.10. Assume Condition B. Let $v(\xi)$ be that in Proposition 4.9. Then $v(\xi)$ can be holomorphically extensible to $\Omega=\{0<|\xi|<r\} \cup S^{*}\left(\theta_{0}, \delta_{0}\right)$ such that $|v(\xi)| \leq A|\xi|^{1-p} e^{c|\xi|^{p}}$.

We can show the holomorphic extension of $v(\xi)$ to an infinete sector $S^{*}\left(\theta_{0}, \delta_{0}\right)$ and $v(\xi) \in \operatorname{Exp}\left(p, S^{*}\right)$ in the same way as the case $\sum_{i=1}^{m} a_{i}=0$ in [5]. Hence we refer to [5] 
for the details.

Theorem 4.2 for case (2) follows from Theorem 4.10. By Remark 2.4 we may assume $f^{(n)}(0)=0$ for $0 \leq n \leq p+n_{0}$ and $u(z)=\sum_{n=n_{0}+1}^{\infty} c_{n} z^{n} \in \mathbb{C}[[z]]$ for a large $n_{0}$. Let $v(\xi) \in \mathcal{O}\left(\{0<|\xi|<r\} \cup S^{*}\left(\theta_{0}, \delta_{0}\right)\right)$ be the solution of (4.9) in Theorem 4.10. Then we can define as case $\mathscr{A}(0) \neq 0$

$$
U(z)=\int_{0}^{\infty e^{i \theta_{0}}} \exp \left(-\left(\frac{\xi}{z}\right)^{p}\right) v(\xi) d \xi^{p},
$$

which is a solution of (2.4) and $p$-Borel summable in the direction $\theta_{0}$, and $U(z) \underset{p}{\sim} u(z)$.

Finally we give a simple example. Let

$$
\begin{aligned}
& u(z)+u\left(\frac{z}{\sqrt[p]{1-z^{p}}}\right)=\frac{z}{1-z}, \\
& \widehat{f}(\xi)=\sum_{n=1}^{\infty} \frac{\xi^{n-p}}{\Gamma(n / p)} .
\end{aligned}
$$

Then $p$-Borel transform of $z /(1-z)$ is $\widehat{f}(\xi)$ and

$$
u(z)=\int_{0}^{\infty e^{i \theta}} \frac{e^{-(\xi / z)^{p}} \widehat{f}(\xi)}{1+e^{\xi^{p}}} d \xi^{p} \quad\left(\frac{\pi}{2}<p \theta<\frac{3 \pi}{2}\right)
$$

and $\exp (2 n+1) \pi i / z^{p}(n \in \mathbb{Z})$ are homogeneous solutions.

\section{Estimates of coefficients of solutions of formal power series.}

In this section we give a proof of Theorem 2.3. The following lemma is given in [5].

LEMMA 5.1. Let $\psi(z)$ be holomorphic in a neighborhood of $z=0$ such that $\psi(z)=$ $1+\sum_{j=p}^{\infty} b_{j} z^{j}$ for $p \geq 1$. Define constants $\left\{B_{k, \ell}\right\}$ by $\psi(z)^{k}=\sum_{\ell=0}^{\infty} B_{k, \ell} z^{\ell}$ for each $k \geq 1$. Then there exists a positive constant $B_{0}$ such that

$$
\left|B_{k, \ell}\right| \leq B_{0}^{\ell} \frac{(k+\ell)^{(k+\ell) / p}}{k^{k / p} \ell^{\ell / p}} .
$$

Since $B_{k, 0}=1$, the notation $\ell^{\ell / p}$ means 1 for $\ell=0$. We have

Proposition 5.2. Assume $\mathscr{A}(0) \neq 0$. Let $u(z)=\sum_{n=1}^{\infty} c_{n} z^{n} \in \mathbb{C}[[z]]$ be a formal solution of (2.4). If $a_{i}(z)(1 \leq i \leq m), f(z) \in \mathbb{C}_{\{1 / p\}}[[z]]$, then there exist positive constants $M$ and $C$ such that

$$
\left|c_{n}\right| \leq M C^{n} n^{n / p} .
$$

Proof. The coefficients $\left\{c_{n}\right\}_{n \geq 1}$ are determined by

$$
\mathscr{A}(0) c_{n}+\sum_{i=1}^{m}\left(\sum_{\substack{j+k+\ell=n \\ k<n}} a_{i, j} c_{k} B_{k, \ell}^{i}\right)=f_{n}
$$


Since $a_{i}(z)=\sum_{j=0}^{\infty} a_{i, j} z^{j}, f(z)=\sum_{j=1}^{\infty} f_{j} z^{j} \in \mathbb{C}_{\{1 / p\}}[[z]]$, there exist constants $A, C_{0}$ and $F$ such that $\left|a_{i, j}\right| \leq A C_{0}^{j} \Gamma(j / p+1)$ and $\left|f_{n}\right| \leq C_{0}^{n} \Gamma(n / p+1)$. We may assume $\left|f_{n}\right| \leq C_{0}^{\prime n} n^{n / p}(n \geq 1)$. We show (5.2) by induction. Since $c_{1}=f_{1} / \mathscr{A}(0)$, by taking $M \geq 2 /|\mathscr{A}(0)|$ and $C>C_{0}^{\prime}$, we have (5.2) for $n=1$. Assume that (5.2) holds for $1 \leq k \leq n-1$. It follows from Lemma 5.1 and Stirling's formula that there exists $C_{1}>0$ such that for $j+k+\ell=n$

$$
\begin{aligned}
\left|a_{i, j} c_{k} B_{k, \ell}^{i}\right| & \leq A C_{0}^{j} \Gamma\left(\frac{j}{p}+1\right) M C^{k} k^{k / p} B_{0}^{\ell} \frac{(\ell+k)^{(k+\ell) / p}}{k^{k / p} \ell^{\ell / p}} \\
& \leq M A C^{k} B_{0}^{\ell} C_{1}^{j} \frac{(j+k+\ell)^{(j+k+\ell) / p}}{\ell^{\ell / p}} \\
& =M A C^{n}\left(\frac{B_{0}}{C}\right)^{\ell}\left(\frac{C_{1}}{C}\right)^{j} \frac{n^{n / p}}{\ell^{\ell / p}} .
\end{aligned}
$$

Take $C>B_{0}, C_{1}$ so large that $A m \sum_{\ell+j \geq 1}\left(B_{0} / C\right)^{\ell}\left(C_{1} / C\right)^{j} \frac{1}{\ell^{\ell / p}} \leq|\mathscr{A}(0)| / 2$ holds. Then

$$
\begin{aligned}
\sum_{\substack { i=1 \\
\begin{subarray}{c}{j+k+\ell=n \\
1 \leq k<n{ i = 1 \\
\begin{subarray} { c } { j + k + \ell = n \\
1 \leq k < n } }\end{subarray}}^{m}\left|a_{i, j} c_{k} B_{k, \ell}^{i}\right| & \leq M A m C^{n} n^{n / p} \sum_{\ell+j \geq 1}\left(\frac{B_{0}}{C}\right)^{\ell}\left(\frac{C_{1}}{C}\right)^{j} \frac{1}{\ell^{\ell / p}} \\
& \leq \frac{M|\mathscr{A}(0)|}{2} C^{n} n^{n / p} .
\end{aligned}
$$

Hence

$$
\left|c_{n}\right| \leq \frac{M}{2} C^{n} n^{n / p}+\frac{C_{0}^{\prime n} n^{n / p}}{|\mathscr{A}(0)|} \leq \frac{M}{2}\left(C^{n}+C_{0}^{\prime n}\right) n^{n / p} \leq M C^{n} n^{n / p} .
$$

Proposition 5.3. Assume $\mathscr{A}(0)=\cdots=\mathscr{A}^{(p-1)}(0)=0$ and $\quad \sum_{i=1}^{m} a_{i}(0) b_{i, p} \neq$ 0 . Let $u(z)=\sum_{n=1}^{\infty} c_{n} z^{n} \in \mathbb{C}[[z]]$ be a formal solution of $(2.4)$. If $a_{i}(z)(1 \leq i \leq m)$, $f(z) \in \mathbb{C}_{\{1 / p\}}[[z]]$, then there exist positive constants $M$ and $C$ such that

$$
\left|c_{n}\right| \leq M C^{n} n^{n / p}
$$

Proof. From the assumption $\mathscr{A}^{(p)}(0) / p !+n\left(\sum_{i=1}^{m} a_{i}(0) b_{i, p}\right) \neq 0$ for $n \geq n_{0}\left(n_{0} \in\right.$ $\mathbb{Z}_{\geq 0}$ ), we may assume by Remark 2.4 that $f(z)=\sum_{n=n_{0}+p+1}^{\infty} f_{n} z^{n} \in \mathbb{C}_{\{1 / p\}}[[z]]$ with $\left|f_{p+n}\right| \leq C_{0}^{\prime n} n^{n / p+1}$ and $u(z)=\sum_{n=n_{0}+1}^{\infty} c_{n} z^{n}$. The coefficients are determined by

$$
\left(\frac{\mathscr{A}^{(p)}(0)}{p !}+n\left(\sum_{i=1}^{m} a_{i, 0} b_{i, p}\right)\right) c_{n}+\sum_{i=1}^{m}\left(\sum_{\substack{j+k+\ell=n+p \\ k<n}} a_{i, j} c_{k} B_{k, \ell}^{i}\right)=f_{n+p} .
$$

We show (5.4) by induction. Put $A_{0}(n)=\mathscr{A}^{(p)}(0) / p !+n\left(\sum_{i=1}^{m} a_{i, 0} b_{i, p}\right)$. Then there exists $A^{\prime}>0$ such that $\left|A_{0}(n)\right| \geq A^{\prime} n$ for $n \geq n_{0}+1$. Since $c_{n_{0}+1}=f_{n_{0}+p+1} / A_{0}\left(n_{0}+1\right)$,

$$
\left|c_{n_{0}+1}\right| \leq \frac{F_{0}}{A^{\prime}\left|n_{0}+1\right|}, \quad F_{0}=C_{0}^{\prime n_{0}+1}\left(n_{0}+1\right)^{\left(n_{0}+1\right) / p+1} .
$$


By taking $M \geq 2 / A^{\prime}$ and $C \geq C_{0}^{\prime}$, we have (5.4) for $n=n_{0}+1$. Assume that (5.4) holds for $n_{0}+1 \leq k \leq n-1$. Then there are constants $A, C_{0}, C_{1}>0$ such that for $j+k+\ell=n+p$

$$
\begin{aligned}
\left|a_{i, j} c_{k} B_{k, \ell}^{i}\right| & \leq A C_{0}^{j} \Gamma\left(\frac{j}{p}+1\right) M C^{k} k^{k / p} B_{0}^{\ell} \frac{(k+\ell)^{(k+\ell) / p}}{k^{k / p} \ell^{\ell / p}} \\
& \leq M A C^{k} B_{0}^{\ell} C_{1}^{j} \frac{(j+k+\ell)^{(j+k+\ell) / p}}{\ell^{\ell / p}} \\
& \leq M A C^{n} n^{n / p+1}\left(\frac{B_{0}}{C}\right)^{\ell-p}\left(\frac{C_{1}}{C}\right)^{j} \frac{B_{0}^{p}}{\ell^{\ell / p}}\left(\frac{n+p}{n}\right)^{n / p+1} \\
& \leq M A A_{1} C^{n} n^{n / p+1} B_{0}^{p}\left(\frac{B_{0}}{C}\right)^{\ell-p}\left(\frac{C_{1}}{C}\right)^{j} \frac{1}{\ell^{\ell / p}}\left(A_{1}=\sup _{n}\left(1+\frac{p}{n}\right)^{1+n / p}\right) .
\end{aligned}
$$

By taking $C$ so large that

$$
A A_{1} m B_{0}^{p} \sum_{\{\ell+j>p\}}\left(\frac{B_{0}}{C}\right)^{\ell-p}\left(\frac{C_{1}}{C}\right)^{j} \frac{1}{\ell^{\ell / p}} \leq \frac{A^{\prime}}{2},
$$

we have

$$
\begin{aligned}
\sum_{i=1}^{m} \sum_{\substack{(j, k, \ell) ; \\
j+k+\ell=n+p, \ell+j>p}}\left|a_{i j} c_{k} B_{k, \ell}^{i}\right| & \leq M A A_{1} m C^{n} n^{1+n / p} B_{0}^{p} \sum_{\{\ell+j>p\}}\left(\frac{B_{0}}{C}\right)^{\ell-p}\left(\frac{C_{1}}{C}\right)^{j} \frac{1}{\ell^{\ell / p}} \\
& \leq A^{\prime} M C^{n} \frac{n^{1+n / p}}{2} .
\end{aligned}
$$

Thus we have by $\left|\left(\mathscr{A}^{(p)}(0) / p !\right)+n \sum_{i=1}^{m} a_{i, 0} b_{i, p}\right| \geq A^{\prime} n$

$$
\left|c_{n}\right| \leq \frac{M}{2} C^{n} n^{n / p}+\frac{C_{0}^{\prime n} n^{n / p}}{A^{\prime}} \leq \frac{M}{2}\left(C^{n}+C_{0}^{\prime n}\right) n^{n / p} \leq M C^{n} n^{n / p} .
$$

Theorem 2.3 follows from Propositions 5.2 and 5.3.

\section{References}

[1] W. Balser, From Divergent Power Series to Analytic Functions, Lecture Notes in Math., 1582, Springer, 1994.

[2] W. Balser, Formal power series and linear systems of meromorphic ordinary differential equations, Universitext, Springer, 2000.

[3] W. Balser, B. L. J. Braaksma, J.-P. Ramis and Y. Sibuya, Multisummabilty of formal power series solutions of linear ordinary differential equations, Asymptotic Analysis, 5 (1991), $27-45$.

[4] S. Ōuchi, Multisummability of formal solutions of some linear partial differential equations, J. Differential Equations, 185 (2002), 513-549.

[5] S. Ōuchi, On some functional equation with Borel summable solutions, Funkcial. Ekvac., 58 (2015), 223-251.

[6 ] W. Wasow, Asymptotic Expansions for Ordinary Differential Equations, Dover Publications, New York, 1987. 


\section{Sunao ŌUCHI}

Sophia University

Tokyo Japan

E-mail: s_ouchi@sophia.ac.jp 